Article

\title{
Flavonoid Mixture Inhibits Mycobacterium tuberculosis Survival and Infectivity
}

\author{
Ruoqiong Cao ${ }^{1, \dagger}{ }^{\text {, Garrett Teskey }}{ }^{1,+}{ }^{\dagger}$, Hicret Islamoglu ${ }^{2, \dagger}{ }^{\text {, Myra Gutierrez }}{ }^{1}$, Oscar Salaiz ${ }^{1}$, \\ Shalok Munjal ${ }^{1}$, Marcel P. Fraix ${ }^{3}$, Airani Sathananthan ${ }^{4}$, David C. Nieman ${ }^{5}$ and \\ Vishwanath Venketaraman $1, *$ (D)
}

1 Department of Basic Medical Sciences, College of Osteopathic Medicine of the Pacific, Western University of Health Sciences, Pomona, CA 91766-1854, USA; rcao@westernu.edu (R.C.); gteskey@westernu.edu (G.T.); myra.gutierrez@westernu.edu (M.G.); oscar.salaiz@westernu.edu (O.S.); shalok.munjal@westernu.edu (S.M.)

2 Western University of Health Sciences College of Dental Medicine, Pomona, CA 91766-1854, USA; hislamoglu@westernu.edu

3 Departments of Physical Medicine and Rehabilitation and Neuromusculoskeletal Medicine/Osteopathic Manipulative Medicine, Western University of Health Sciences College of Osteopathic Medicine of the Pacific, Pomona, CA 91766-1854, USA; mfraix@westernu.edu

4 Department of Internal Medicine, Western University of Health Sciences College of Osteopathic Medicine of the Pacific, Pomona, CA 91766-1854, USA; asathananthan@westernu.edu

5 Department of Health and Exercise Science, Appalachian State University, North Carolina Research Campus, Kannapolis, NC 28081, USA; niemandcasu@gmail.com

* Correspondence: vvenketaraman@westernu.edu; Tel.: +1-909-706-3736

+ Authors contributed equally to this work.

Received: 12 January 2019; Accepted: 22 February 2019; Published: 28 February 2019

\begin{abstract}
Background: Flavonoids have been shown to exert anti-pathogenic potential, but few studies have investigated their effects on Mycobacterium tuberculosis $(M t b)$ infectivity. We hypothesized that a flavonoid mixture would have a favorable influence on cell death and the resolution of $M t b$ infection in THP-1 macrophages and in granulomas derived from both healthy participants and those with type 2 diabetes mellitus (T2DM). METHODS: THP-1 macrophages, and in vitro granulomas from healthy participants $(N=8)$ and individuals with T2DM $(N=5)$ were infected with $M t b$. A mixed flavonoid supplement (MFS) at a concentration of $0.69 \mathrm{mg}$ per $\mathrm{ml}$ was added as treatment to $\mathrm{M} t b$ infected THP-1 macrophages and granulomas for 8 to 15 days. RESULTS: MFS treatment significantly reduced the intracellular $M t b$ survival, increased cell density, aggregation, and granuloma formation, and increased glutathione (GSH) levels. IL-12 and IFN- $\gamma$ levels tended to be higher and IL-10 lower when $M t b$ infected THP-1 macrophages and granulomas obtained from healthy subjects were treated with MFS compared to control. CONCLUSIONS: MFS treatment exerted a strong influence against $M t b$ infectivity in THP-1 macrophages and in granulomas including antimycobacterial effects, GSH enrichment, cytokine regulation, and augmented granuloma formation. Our data support the strategy of increased flavonoid intake for managing tuberculosis.
\end{abstract}

Keywords: Mycobacterium tuberculosis; Mtb; TB; flavonoids; immunomodulation; glutathione; T2DM; polyphenols

\section{Introduction}

Mycobacterium tuberculosis (Mtb), the etiological agent which causes tuberculosis (TB), is the leading cause of infectious death worldwide. $M t b$ is transmitted from person to person via aerosol droplets typically by means of coughing. In 2017, there was an estimated 10.0 million new cases of TB reported, and 1.3 million deaths worldwide among human immunodeficiency virus (HIV)-negative 
individuals [1]. Typical treatment of active TB includes an extensive antibiotic regimen of isoniazid, rifampin, ethambutol and pyrazinamide, which have specific mechanisms of action in combating $M t b$ infection. However, due to the emergence of antibiotic-resistant strains of $M t b$ treatment is becoming more problematic [2]. Therefore, many new treatment options are being explored as possible alternatives or adjunctive therapies to combat this devastating disease. There is currently an urgency to find new treatment procedures for $M t b$ infections due to the emergence of multi-drug resistant strains of $M t b[1]$.

Despite substantial advancements attended in this field, diabetic patients are still considerably more likely to contract TB than nondiabetic individuals [3]. The number of people with diabetes has risen from 108 million in 1980 to 422 million in 2014. Four out of five adults with diabetes live in lowand middle-income countries where the prevalence of TB and other infectious diseases is high. In 2016, an estimated 1.6 million deaths were directly caused by diabetes [4]. It is commonly accepted that diabetes decreases the effectiveness of cell mediated immunity thus making these individuals more vulnerable to $M t b$ infection, but the exact mechanism for $M t b$ susceptibility in individuals with T2DM is not entirely well understood [3].

Polyphenols are a structural class of organic plant-based compounds, characterized by the presence of multiple units and responsible for many natural food pigmentations. Nearly half of the polyphenols are flavonoids, which can be further divided into flavan-3-ols, flavanones, flavones, isoflavones, flavonols, anthocyanins and proanthocyanidins [5]. The Phenol-Explorer database on polyphenol content in foods contains values for 500 different polyphenols in over 400 foods in the human diet (http:/ / phenol-explorer.eu/). Recommendations for dietary polyphenol and flavonoid intake have not yet been established. In Europe, the average dietary polyphenol intake has been estimated at $1.187 \mathrm{~g} /$ day with coffee, tea, fruits, and wine as the principal sources [6]. Nearly all ingested polyphenols pass through the small intestine unabsorbed and reach the colon where bacterial degradation produces smaller phenolics that can be reabsorbed into the circulation after undergoing phase 2 conjugation in the liver [7]. The gut-derived phenolics circulate throughout the body exerting a variety of bioactive effects. Studies support a robust relationship between high dietary polyphenol intake and reduced risks for overall mortality and several chronic diseases, acute respiratory illness, inflammation, and oxidative stress [7-9]. Two human studies suggest an inverse relationship between regular intake of flavonoid-rich tea beverages and risk for TB $[10,11]$.

Cell culture studies support a strong anti-pathogenic influence from selected flavonoids including quercetin, anthocyanins, and flavan-3-ols [12-14]. For example, serum samples collected from athletes that contained metabolites from blueberry and green tea ingestion protected cells from killing by the vesicular stomatitis virus [12]. Limited evidence indicates that epigallocatechin-3-gallate (EGCG) and quercetin inhibit $M t b$ growth and survival within human macrophages $[13,14]$. We sought to extend these findings by measuring the influence of a flavonoid mixture (quercetin, green tea flavan-3-ols, bilberry anthocyanins) on cell death and resolution of infection in a variety of $M t b$ infected cells.

\section{Results}

\subsection{Survival of Mtb Erdman Subsequent to MFS Treatment}

The direct antimycobacterial effects of the MFS was determined during the log phase Mtb growth in 7H9 media. After eight days of incubation, a twofold decrease in bacterial quantity was measured when $M t b$ was treated with the MFS compared to sham treatment (Figure 1). 


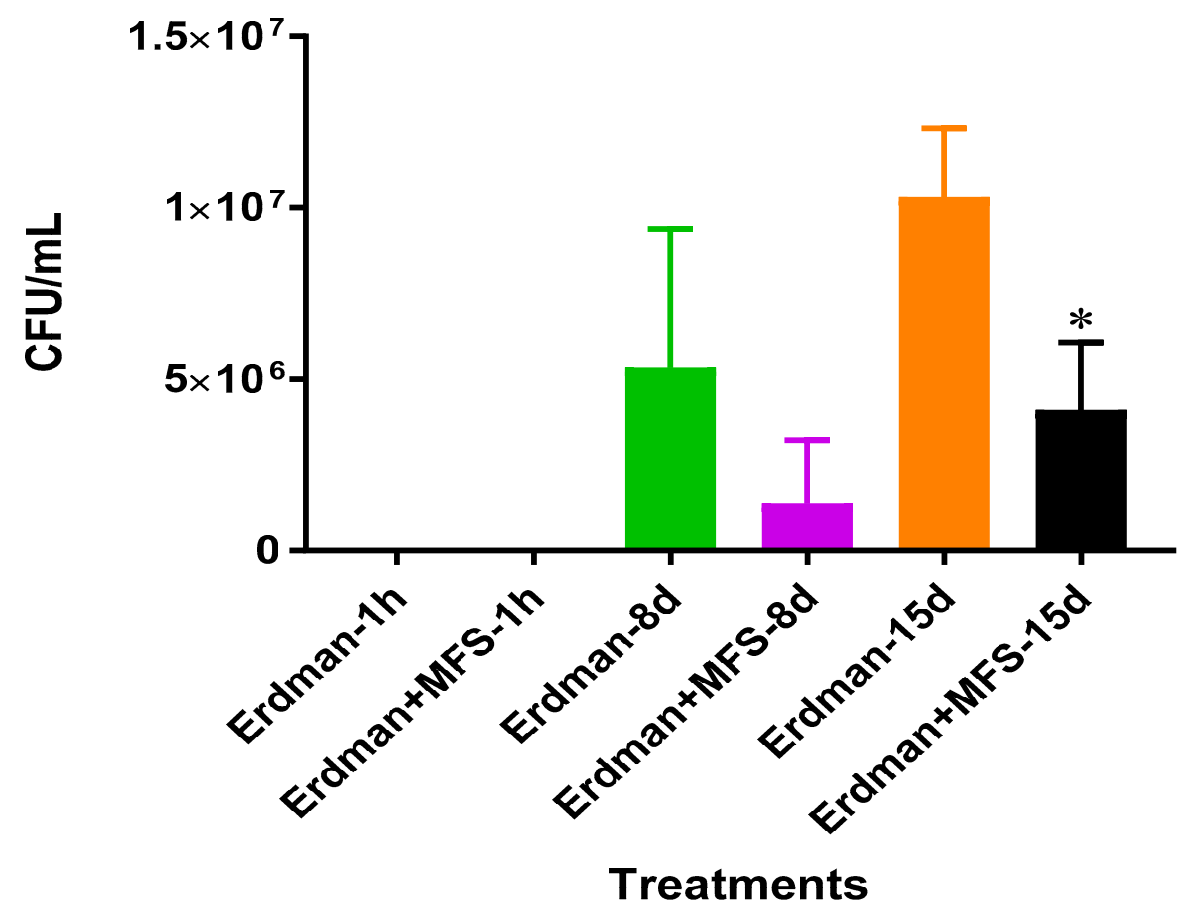

Figure 1. Growth of Mtb Erdman in 7H9 containing MFS. Experiments were performed in order to determine direct mycobacterial effects of each treatment. Data represent means \pm SE from 1 trial and plating it multiple times. ${ }^{*} p<0.05$ when comparing samples to their respective controls.

\subsection{Hematoxylin and Eosin Staining of THP-1 Cells}

Hematoxylin and Eosin (H\&E) staining of THP-1 macrophages showed an increase in cell quantity after MFS treatment versus the sham-treated after 12 days of infection (Figure 2). These data indicate that THP-1 macrophage viability was enhanced after MFS treatment.

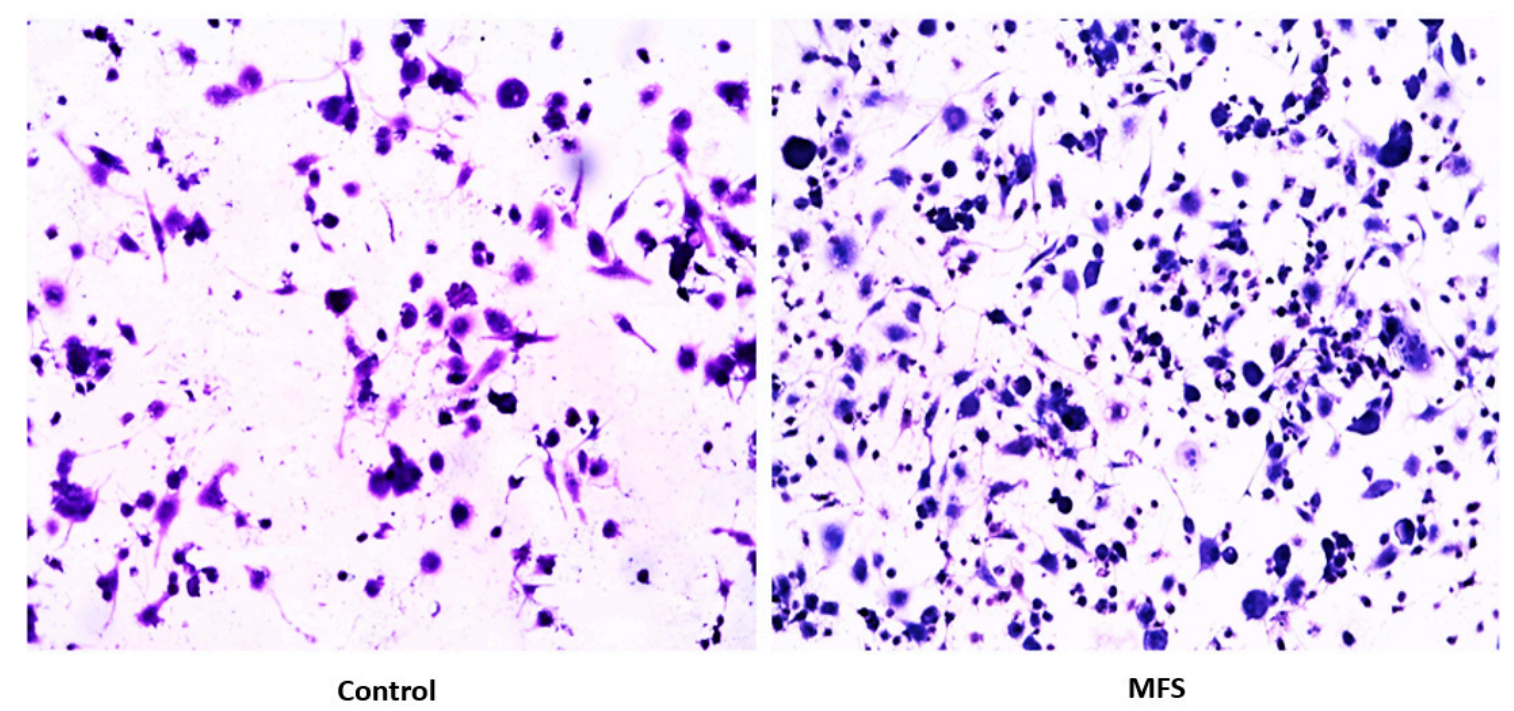

Figure 2. Hematoxylin and Eosin staining of untreated and MFS-treated samples of THP-1 cells. Microscopy work was done under $100 \times$. 


\subsection{Intracellular Survival of Mtb Erdman from THP-1 Cells}

The bacterial quantity of $M t b$ Erdman was approximately 2.5 times lower when THP-1 cells were treated with MFS versus no treatment after 12 days (Figure 3). These data indicate that MFS treated THP-1 cells exhibited an enhanced ability to eliminate and/or contain the Mtb infection.

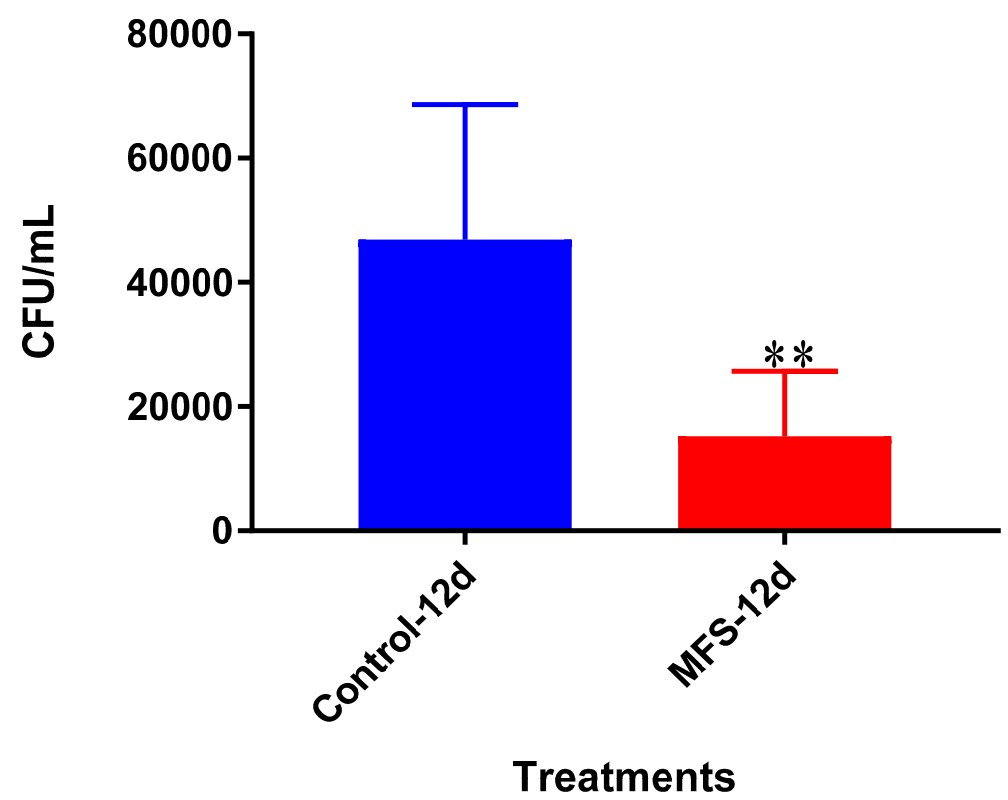

Figure 3. Survival of Mtb Erdman inside untreated and MFS-treated THP-1 macrophages. Data represent means \pm SE from 6 trials and multiple plating times. ${ }^{* *} p<0.005$ when comparing MFS-treated samples to untreated samples at 12 days.

\subsection{Levels of IL-10 from THP-1 cell Supernatant}

Although not statistically significant, IL-10 levels were diminished when THP-1 cells were treated with MFS compared to the sham-treated infected-control (Figure 4).

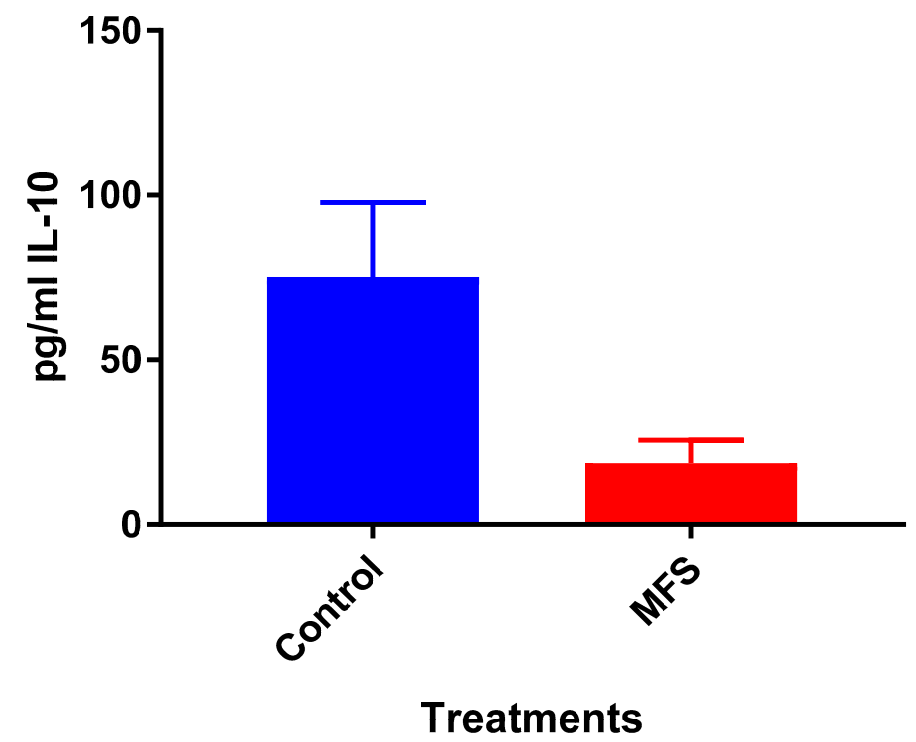

Figure 4. Levels of IL-10 in untreated and MFS-treated THP-1 cells. Assay of IL-10 was performed using an enzyme-linked immunosorbent assay (ELISA) Ready-Set-Go kit from eBioscience. Data represent means \pm SE from 6 trials. 


\subsection{Levels of Total GSH from Mtb Erdman Infected THP-1 cell Lysate}

Mtb infected THP-1 cells displayed a statistically significant increase in GSH levels after MFS treatment compared to the control category (Figure 5).

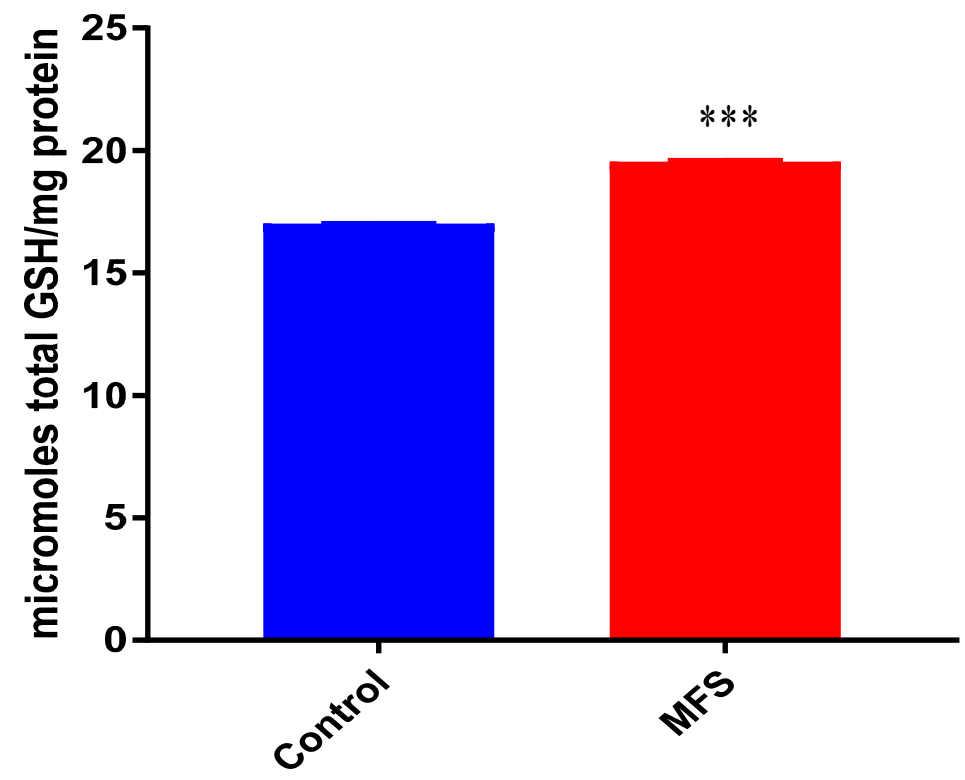

Treatments

Figure 5. Levels of total Glutathione (GSH) in untreated and MFS-treated THP-1 cells. Corrections were made to total protein measured by BCA Protein Assay Kit from Thermo Scientific. Data represent means \pm SE from 6 trials. ${ }^{* * *} p<0.0005$ when comparing GSH levels from control and MFS treated groups.

\subsection{Hematoxylin and Eosin Staining of Human Granulomas from Healthy Individuals}

H\&E staining of $M t b$ infected peripheral blood mononuclear cells (PBMCs) illustrates an increase in cell survival with MFS administration versus sham treatment after 15 days of infection (Figure 2), as well as an increase in cell density/aggregation and granuloma formation (Figure 6).

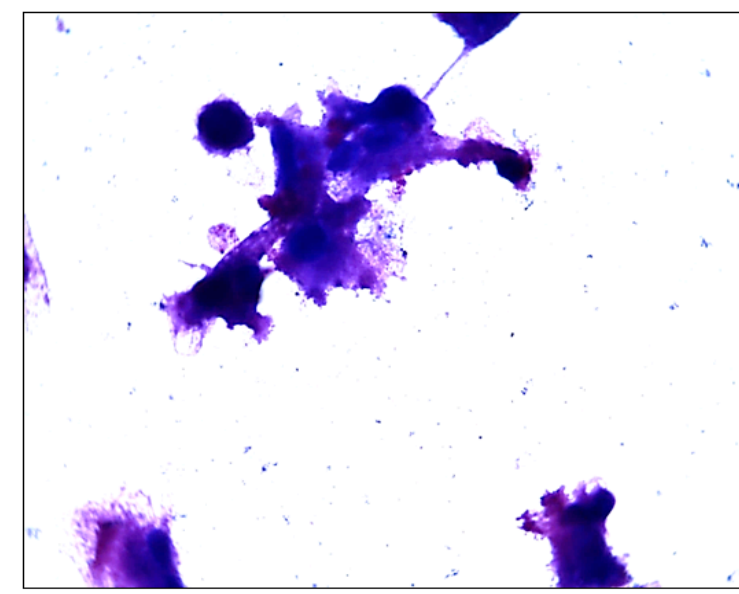

Control

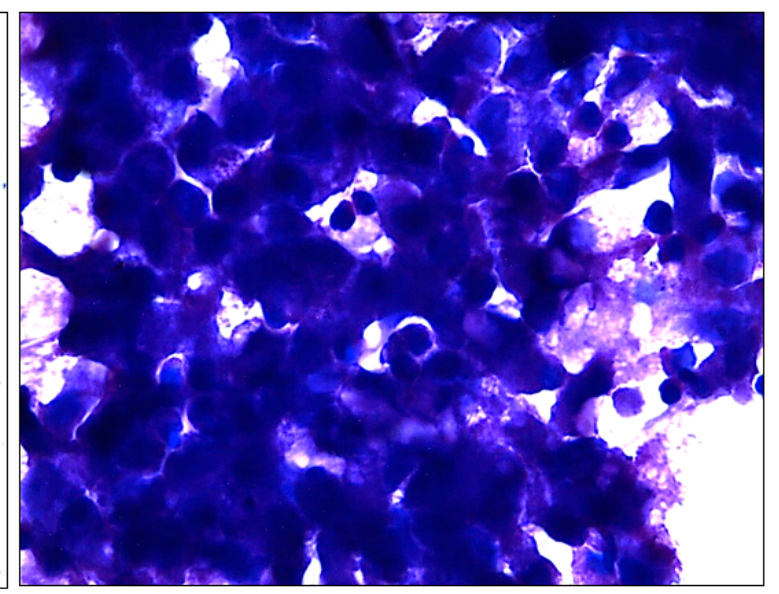

MFS

Figure 6. Hematoxylin and Eosin staining of untreated and MFS-treated human granulomas from healthy subjects. Microscopy work was done with a light microscope at $100 \times$ magnification under oil immersion. 
2.7. Intracellular Survival of Mtb Erdman from the in vitro Granulomas of PBMCs Drawn from Healthy Subjects

The bacterial quantity of $M t b$ Erdman was approximately twofold lower from the granulomas formed from the PBMCs of healthy subjects administered with MFS versus the sham-treated after 15 days post infection (Figure 7 ).

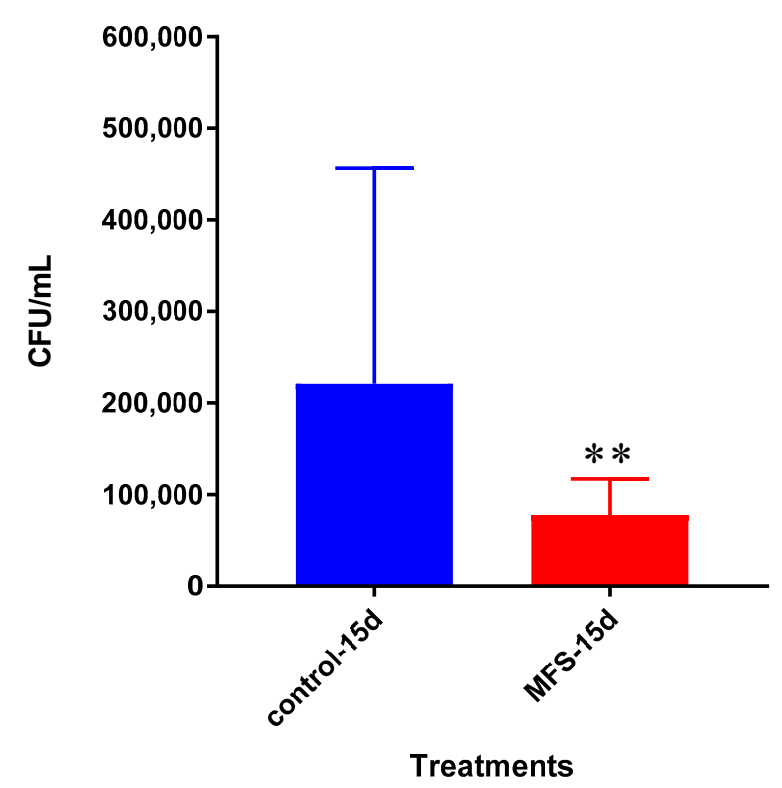

Figure 7. Survival of $M t b$ Erdman inside untreated and MFS-treated granulomas from healthy subjects. Data represent means \pm SE from eight healthy individuals. ${ }^{* *} p<0.005$ when comparing samples at 15 days.

\subsection{Levels of Total GSH from Healthy Subjects}

$M t b$ infected granulomas from PBMCs of healthy subjects showed a 2.5-fold greater increase in intracellular GSH levels after MFS versus sham treatment (Figure 8).

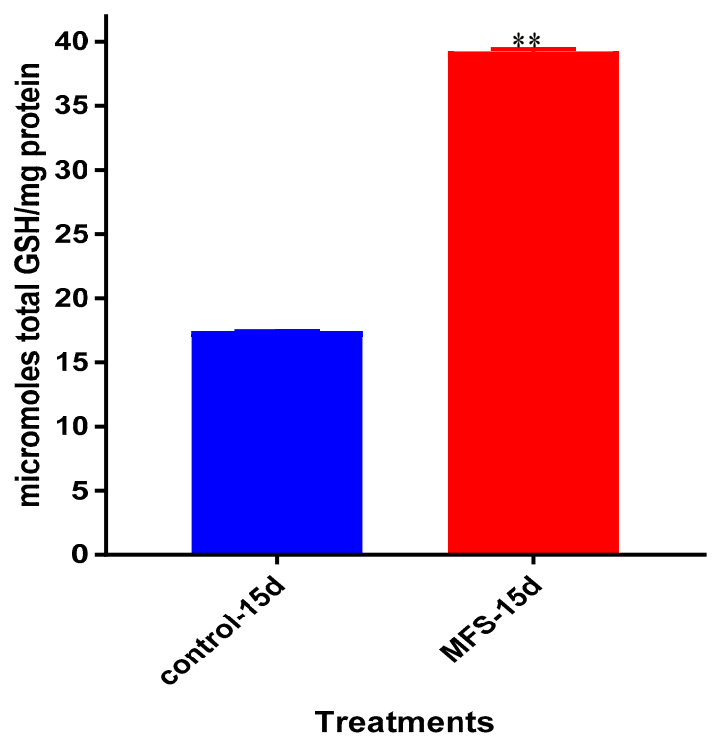

Figure 8. Levels of total GSH in untreated and MFS-treated granulomas from healthy subjects. Data represent means \pm SE from eight healthy individuals. ${ }^{* *} p<0.005$ when comparing GSH levels in control and MFS-treated groups. 
2.9. Levels of IL-12, IFN- $\gamma, I L-10$ from the Supernatants of Healthy Subjects

After 15 days of infection, the IL-12 and IFN- $\gamma$ levels tended to be higher, and IL-10 lower when $M t b$ infected granulomas derived from PBMCs obtained from healthy subjects were treated with MFS compared to the sham-treated infected-control (Figures 9-11).

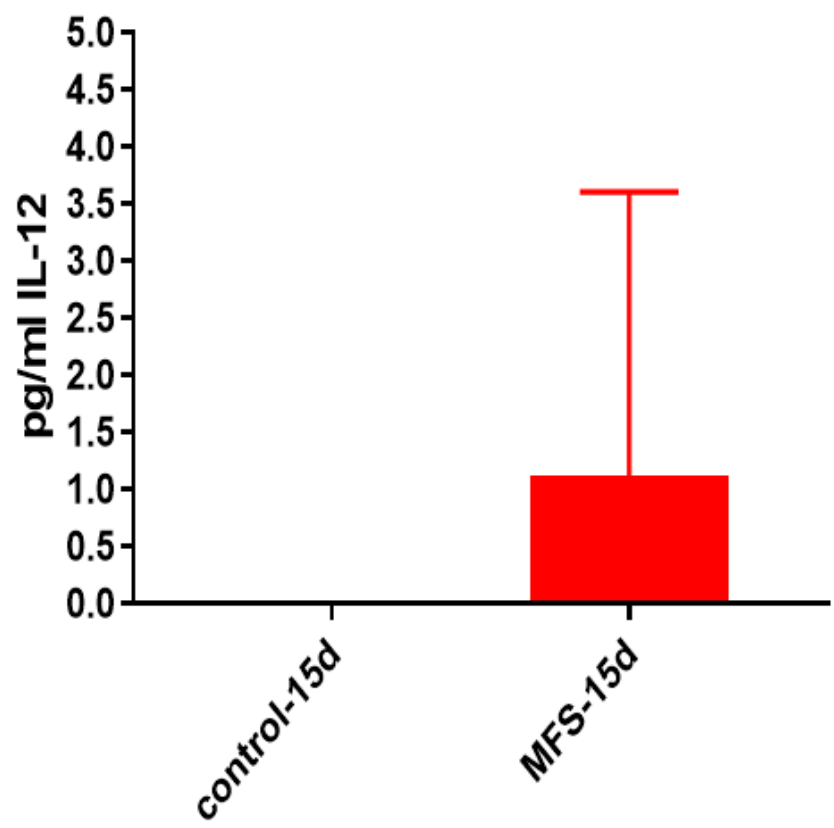

Treatments

Figure 9. Assay of IL-12 in the supernatants from untreated and MFS-treated granulomas from healthy subjects. Assay of IL-12 was performed using an ELISA Ready-Set-Go kit from eBioscience. Data represent means $\pm \mathrm{SE}$ from eight healthy individuals.

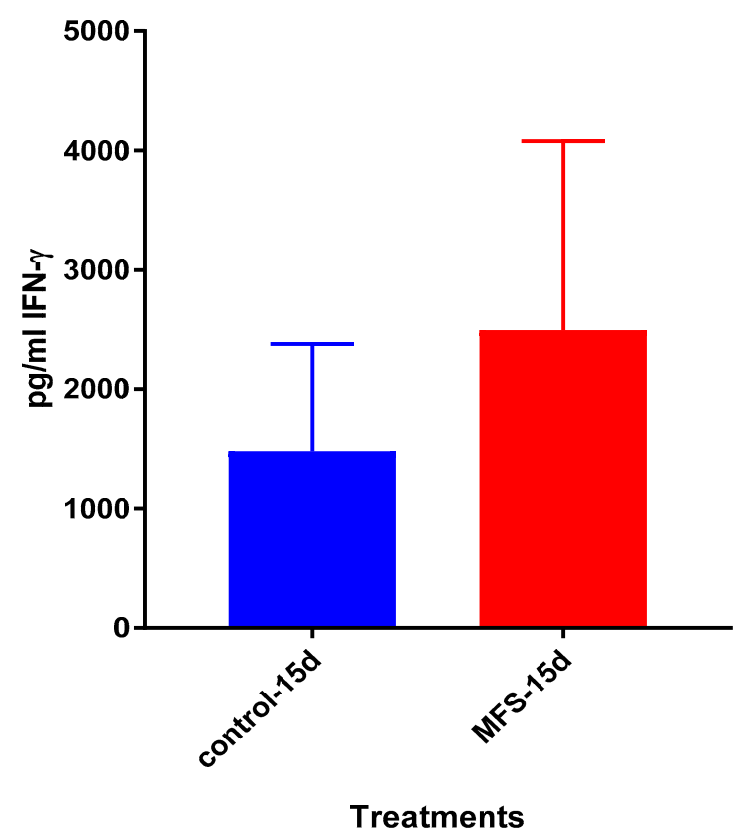

Figure 10. Assay of IFN- $\gamma$ in the supernatants from untreated and MFS-treated granulomas from healthy subjects. Assay of IFN- $\gamma$ was performed using an ELISA Ready-Set-Go kit from eBioscience. Data represent means \pm SE from eight healthy individuals. 


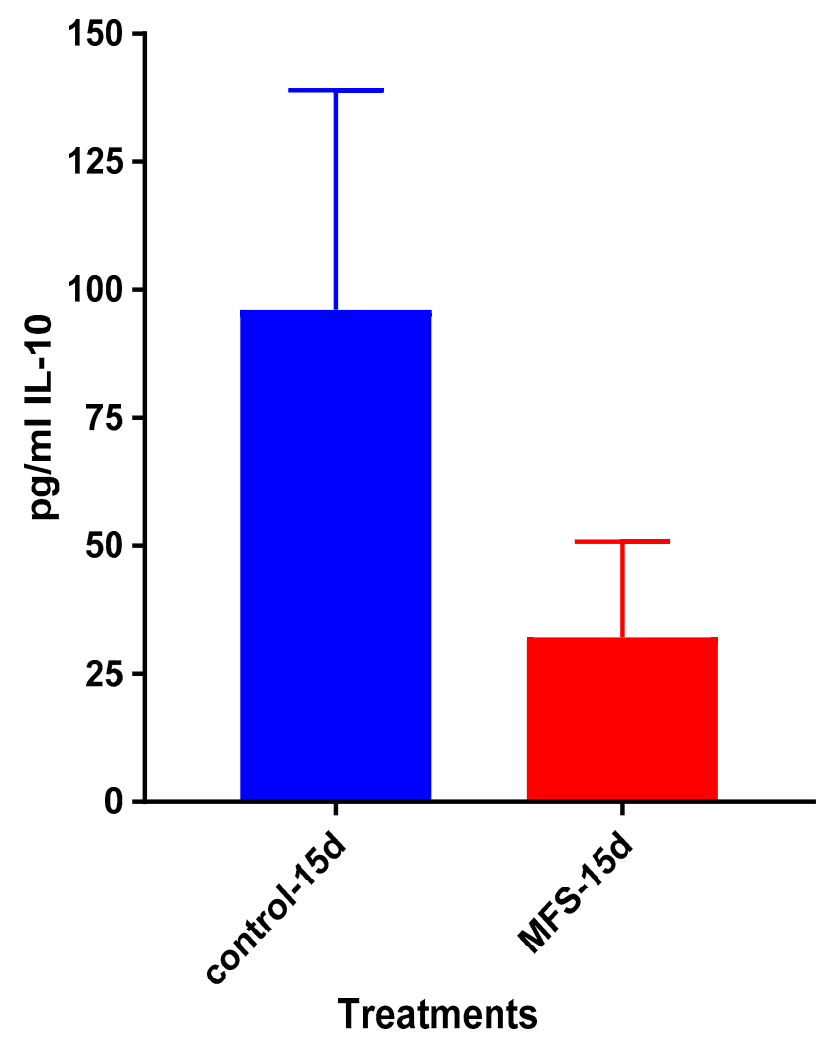

Figure 11. Assay of IL-10 in the supernatants from untreated and MFS-treated granulomas from healthy subjects. Assay of IL-10 was performed using an ELISA Ready-Set-Go kit from eBioscience. Data represent means \pm SE from eight healthy individuals.

\subsection{Hematoxylin and Eosin Staining of Human Granulomas from Individuals with T2DM}

H\&E staining of $M t b$ infected granulomas from individuals with T2DM showed an increase in cell density and aggregation with MFS versus sham treatment after 15 days (Figure 12).

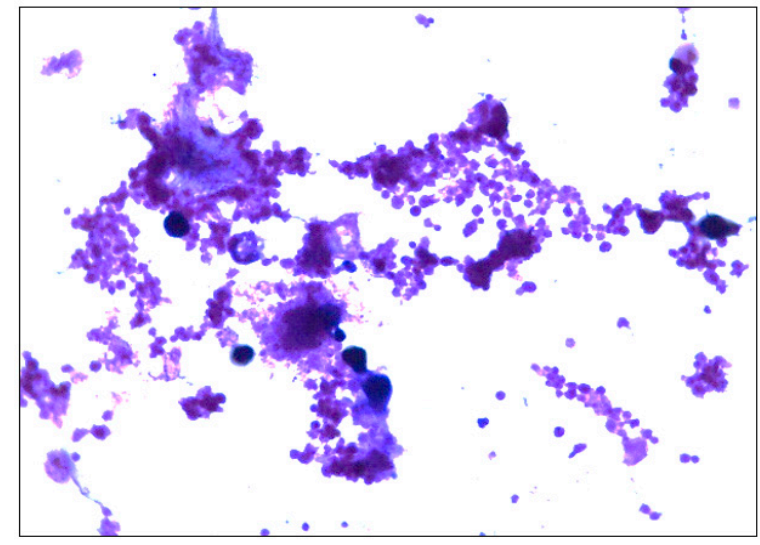

Control

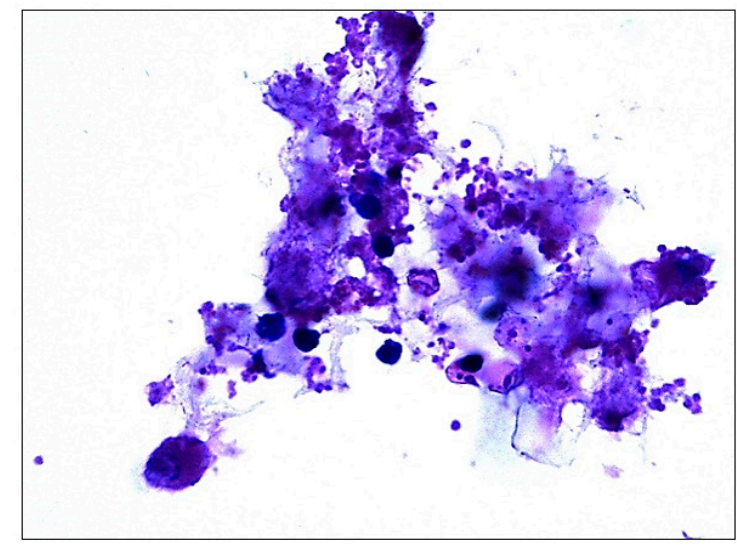

MFS

Figure 12. Hematoxylin and Eosin staining of untreated and MFS-treated human granulomas from individuals with T2DM. Microscopy work was done with a light microscope at $100 \times$ magnification under oil immersion.

\subsection{Survival of Mtb Erdman Inside Granulomas from Individuals with T2DM}

The bacterial quantity of $M t b$ Erdman observed was lower from the granulomas formed from T2DM individuals following MFS administration versus the sham-treated after 15 days (Figure 13). 
The overall bacterial quantities were substantially higher among the T2DM individuals than observed among the healthy study participants (Figure 14).

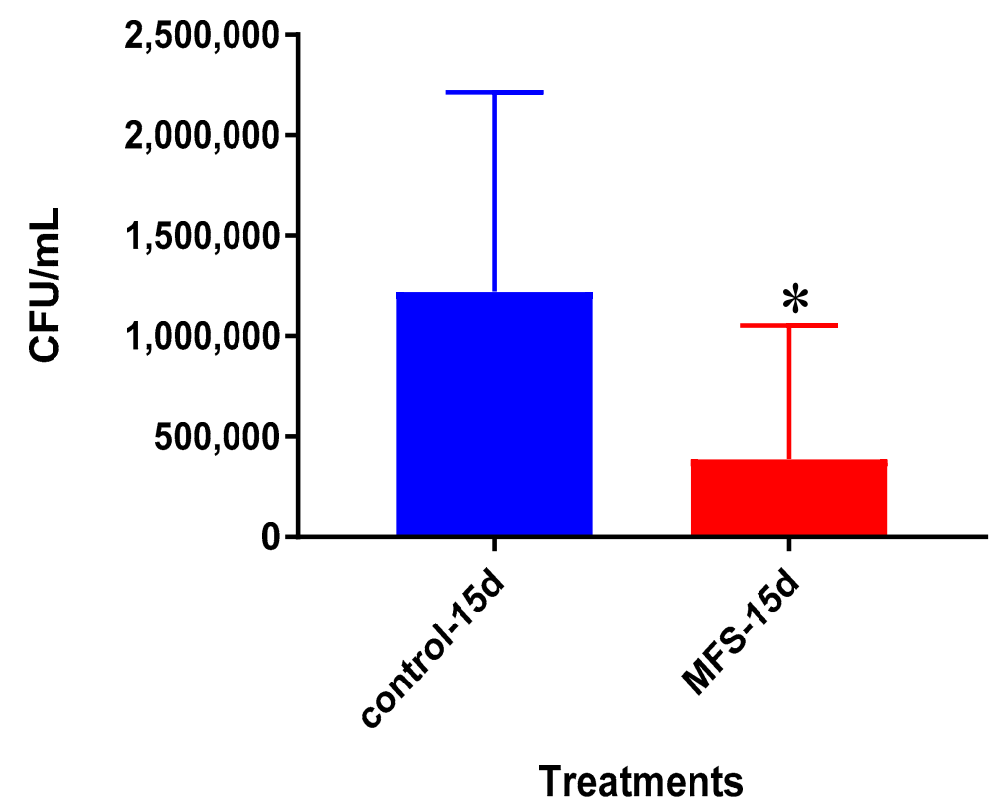

Figure 13. Survival Mtb Erdman inside untreated and MFS-treated granulomas from individuals with T2DM. Data represent means \pm SE from six T2DM individuals. ${ }^{*} p<0.05$ when comparing untreated samples with samples treated with MFS at 15 days.

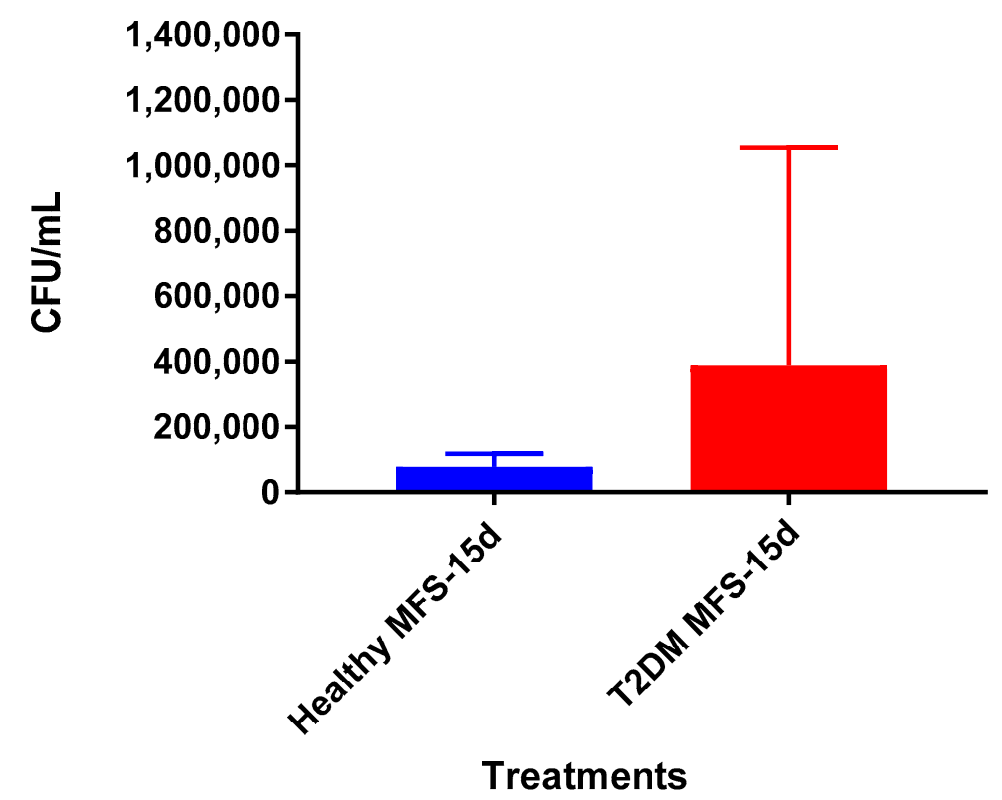

Figure 14. Survival of Mtb Erdman inside MFS-treated granulomas from healthy subjects and individuals with T2DM. Data represent means \pm SE from six T2DM individuals and \pm SE from healthy individuals.

\subsection{Levels of IL-10 from the Supernatants of Individuals with T2DM}

IL-10 levels tended to be lower when $M t b$ infected granulomas from healthy subjects were treated with MFS compared to the sham-treated infected-control after 15 days (Figure 15). Overall, the IL-10 levels in individuals with T2DM were about two-fold higher than observed in the healthy participants (Figure 11). 


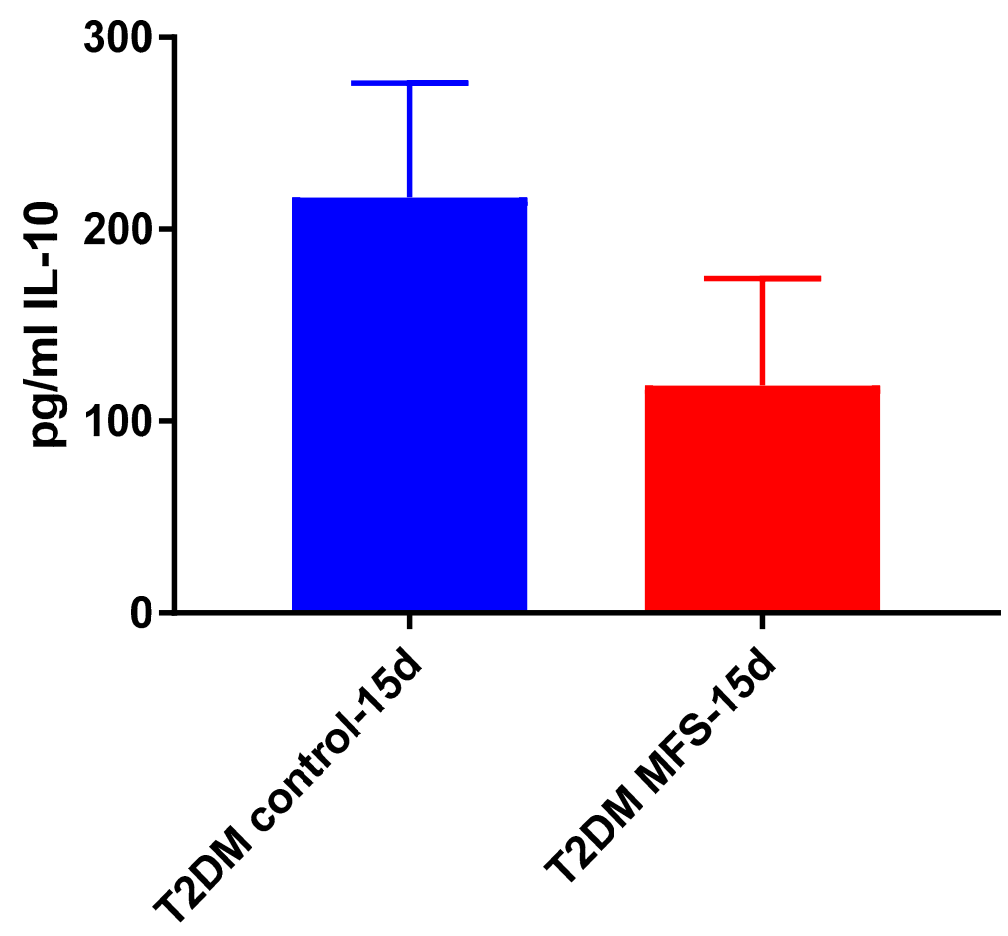

Treatments

Figure 15. Levels of IL-10 in untreated and MFS-treated granulomas from individuals with T2DM. Assay of IL-10 was performed using an ELISA Ready-Set-Go kit from eBioscience. Data represent means \pm SE from six T2DM individuals.

\section{Discussion}

While TB still plagues roughly one third of the world's population, flavonoids may offer an exciting and inexpensive alternative therapy due to their direct antimycobacterial and immunomodulating effects. Following food and beverage ingestion, small amounts of flavonoids are absorbed in the small intestine after sulfate, glucuronide, and/or methyl conjugation [7]. Once in the bloodstream, the conjugated flavonoid metabolites can undergo further phase II metabolism in the liver prior to interactions at the cell level and ultimate urinary excretion. Enzymes at the cell level may deconjugate flavonoid metabolites, allowing the more active aglycones to exert beneficial effects as measured in cell culture studies [7]. The majority of ingested flavonoids pass into the large intestine where they undergo extensive microbial catabolism followed by fecal excretion or reentry into the circulatory system as biotransformed phenolic metabolites [7]. Some studies indicate that following flavonoid supplementation, serum samples containing a mixture of conjugated flavonoid metabolites and colon-derived phenolics exert anti-pathogenic effects [12,15].

We first measured direct bactericidal influences by adding MFS to Mtb suspended in 7H9 broth media. After 15 days (Figure 1), MFS versus control treatment resulted in a reduction in bacterial load (Figure 1). These results demonstrated that flavonoids exerted direct antibacterial action against $M t b$, although we cannot rule out the potential synergistic effects of the added adjuvants caffeine, vitamin C, and omega 3 fatty acids. This result compares favorably with those from Sasikumar et al. [13] who showed that quercetin exhibited $99 \%$ and $56 \%$ inhibition against $M t b \mathrm{H} 37 \mathrm{Rv}$ at $200 \mu \mathrm{g} / \mathrm{mL}$ and $50 \mu \mathrm{g} / \mathrm{mL}$, respectively.

Subsequently, we measured the effects of 12 days of MFS treatment on Mtb infected human macrophages derived from THP-1 monocytes. MFS supplementation compared to the control condition over a 12-day period improved the viability of the human THP-1 cells as depicted by the H\&E imaging, which portrayed a greater cell magnitude (Figure 1). This suggested that $M t b$ infection became 
too vigorous for the THP-1 cells to retain and secure in the absence of MFS prophylactic treatment. This conclusion is further supported by the data represented in Figure 2, which illustrates that after 12 days MFS treatment resulted in a statistically significant bacterial reduction compared to the sham-treated control. These results support the findings of Anand et al. [14] who showed that the green tea flavonoid EGCG inhibited $M t b$ survival within macrophages. This research group showed that EGCG down-regulated host molecule tryptophan-aspartate containing coat protein (TACO) gene transcription, thus influencing phagosome maturation and the capacity to contain $M t b$.

Of the three cytokines measured in this study, IL-10 was most effected by MFS treatment with levels half that of the control condition (Figure 4). IL-10 is an immunosuppressive cytokine which down regulates the expression of Th1 cytokines, NF-kB activity, and macrophage costimulatory molecules [16]. Thus, in the context of a Mtb infection, IL-10's inhibition subsequent to MFS administration is considered to be favorable.

Interestingly, the levels of detectable intracellular GSH were elevated following MFS treatment. Our lab has previously demonstrated that among $M t b$ infected cells, the augmentation of GSH will cause mycobactericidal effects in both a direct and immunomodulating indirect manner through downstream mediators $[17,18]$. Therefore, MFS treatment of THP-1 cells can diminish $M t b$ infectivity in direct and indirect pathways.

The influence of MFS on Mtb infected PBMCs collected from eight healthy individuals was similar to the findings from the THP-1 macrophage assays. The H\&E data showed enhanced PBMC cell viability, cell aggregation/density, and enriched granuloma formation after MFS treatment (Figure 6). This is significant because augmented granuloma development is linked to increased bacterial containment and improved host protection [19]. As with the THP-1 macrophages, the increased immune cell viability observed subsequent to MFS treatment coincided with a strong reduction in bacterial density among the infected PBMCs (Figures 2 and 6). Taken together with the GSH and cytokine data, these results support direct MFS-related mycobactericidal effects and immunomodulation from granuloma formation and the trend for increased IL-12 and IFN- $\gamma$ cytokine production (Figures 8-11). Both IL-12 and IFN- $\gamma$ are prominently involved in immune cell differentiation and activation in response to combating $M t b$ infection, a process linked to granuloma formation [20].

Lastly, we tested the influence of MFS treatment on PBMCs collected from individuals with T2DM, a patient population that is significantly more susceptible to $M t b$ infections [1]. Consistent with findings from the healthy individuals, PBMCs of T2DM patients treated with MFS exhibited more robust cellular aggregations and granuloma formation (Figure 12). Correspondingly, after MFS administration the PBMC bacterial burden was reduced to roughly one-third that of the T2DM infected control (Figure 13). Although the abundance of $M t b$ was significantly diminished with MFS treatment, the quantity of bacteria remaining was still over double that of levels linked to the same experiments in healthy study participants (Figure 14). This demonstrates that while MFS supplementation reduced $M t b$ infectivity in both healthy individuals and those with T2DM, it is not sufficient to cause complete Mtb elimination.

\section{Materials and Methods}

\subsection{THP-1 Cell Culture}

The THP-1 cell line originated from the American Type Culture Collective (ATCC), was cultured in Roswell Park Institute (RPMI) medium (Sigma, St. Louis, MO, USA) with 10\% Fetal bovine serum (FBS-Sigma) and incubated at $37{ }^{\circ} \mathrm{C}$ with $5 \% \mathrm{CO}_{2}$. After $7-12$ days, cells were collected from the flasks, spun at $2000 \mathrm{rpm}$ for $15 \mathrm{~min}$, resuspended in RPMI containing 10\% FBS and counted for cell numbers. THP- 1 cells $\left(2 \times 10^{5} /\right.$ well) were distributed in $0.001 \%$ poly-lysine (Sigma, St. Louis, MO, USA) coated 24-well tissue culture plates and incubated overnight. Differentiation of THP-1 cells to macrophages was achieved by adding PMA (Phorbol 12-myristate 13-acetate-Sigma) at a concentration of $10 \mathrm{ng} / \mathrm{mL}$. 


\subsection{Culture of Mtb Erdmann}

$M t b$ Erdmann expressing green fluorescent protein (GFP) was obtained as a gift from Dr. Selvakumar Subbian at Rutgers New Jersey Medical School, Biomedical and Health Sciences. Mtb Erdmann is similar to H37Rv (standard laboratory strain of $M t b$ ) however it is considered to be more virulent due to its faster doubling time [21]. Mtb Erdmann was used for all experiments conducted in this study, handled inside the biosafety level 3 facility (BSL-3) and cultured in Difco Middlebrook 7H9 broth media supplemented with ADC (Albumin Dextrose Catalase) at $37^{\circ} \mathrm{C}$. Mtb Erdman was processed for infection once the static culture was at the peak logarithmic phase of growth (optical density at $600 \mathrm{~nm}$, between 0.5 and 0.8 ) and subsequently washed and resuspended in sterile $1 \times$ phosphate-buffered saline (PBS). The Mtb Erdman was then processed to disaggregate any clumps by vortexing five times with 3-mm sterile glass beads at $3 \mathrm{~min}$ intervals. Mtb Erdman suspension was then filtered using a $5-\mu \mathrm{m}$ syringe filter to remove any remaining bacterial aggregations. The single cell suspension of now processed $M t b$ was then serially diluted and plated on 7H11 agar (Hi Media, Santa Maria, CA, USA) to determine the bacterial concentration of the processed stock. Aliquots of processed bacterial stocks were then frozen and stored in the cryogenic freezer at $-80^{\circ} \mathrm{C}$. At the time of the experimental trial, the frozen-processed stocks of $M t b$ were thawed and used for the infection.

\subsection{Subject Recruitment}

Study subjects were recruited after obtaining signed informed consent. The protocols for all the studies pertaining to in vitro $M t b$ infection were approved by the Institutional Biosafety committee of Western University of Health Sciences. The protocols for the study involving healthy subjects and participants with T2DM were approved by the Institutional Review Board of Western University of Health Sciences. Healthy adults ( $N=8$, ages 21 to 28 years, no known diseases or medication use) and individuals with T2DM ( $N=5$, ages 51 to 74 years, hemoglobin $\mathrm{A} 1 \mathrm{C}>7 \%)$ were recruited after obtaining signed informed consent. Exclusion criteria included the use of MFS within the last 6 months, or history of chemotherapy treatment within the last year. Other exclusion criteria included women who were currently pregnant, lactating, or had been pregnant within the last 6 months; pregnancy was considered a reason for study termination. After signing the informed consent form, $40 \mathrm{~mL}$ of blood was drawn from each participant.

\subsection{Flavonoids}

The mixed flavonoid supplement (MFS) was prepared by Reoxcyn LLC (Pleasant Grove, UT, USA). Supplement ingredients (US Patent 9,839,624) provided (in one tablet) $82.3 \mathrm{mg}$ total monomeric flavonoids and included $25 \mathrm{mg}$ vitamin C (as ascorbyl palmitate) (Green Wave Ingredients, La Mirada, CA, USA), wild bilberry fruit extract with $16 \mathrm{mg}$ anthocyanins

(FutureCeuticals, Momence, IL, USA), green tea leaf extract with $46 \mathrm{mg}$ total flavan-3-ols (Watson Industries, Inc., Pomona, CA, USA), 26 mg quercetin aglycone (Novel Ingredients, East Hanover, NJ, USA), $26.8 \mathrm{mg}$ caffeine (Creative Compounds, Scott City, MO, USA), and $15 \mathrm{mg}$ omega 3 fatty acids (Novotech Nutraceuticals, Ventura, CA, USA). As previously reported, the tablet contents were analyzed prior to the study for flavonoid content using high-performance liquid chromatography (HPLC) [22]. Thirteen anthocyanins were identified in the bilberry extract including delphinidin, cyanidin, petunidin, peonidin, and malvidin galactosides, glucosides, and arabinsosides. The supplement tablets were analyzed again after 12 weeks and 12 months of storage at room temperature, and the data indicate that all chemical components were stable. As previously described, vitamin C, caffeine, and omega 3 fatty acids were included as adjuvants to improve flavonoid bioactivity [22]. For in vitro administration, the flavonoid tablet $(138 \mathrm{mg})$ was dissolved in $1 \mathrm{~mL}$ of $100 \%$ DMSO. Once dissolved, the solution was diluted with $9 \mathrm{~mL}$ of RPMI. This method brought the stock concentration to $13.8 \mathrm{mg}$ of dissolved flavonoid tablet per $\mathrm{mL}$. We used $25 \mathrm{uL}$ of this solution per each $500 \mathrm{uL}$ well, making the final concentration $0.69 \mathrm{mg}$ of flavonoids per $\mathrm{mL}$. 


\subsection{Determination of the Direct Antimycobacterial Effects of MFS}

To determine the direct effects of MFS in altering the survival of Mtb Erdman, bacteria $\left(6 \times 10^{4}\right.$ /well) were grown in 7H9 media in 24 well tissue culture plates (Corning, NY, USA) in the presence and absence of MFS up to 15 days. Mtb Erdman cultures were then either sham-treated (control) or treated with the MFS at a concentration of $0.69 \mathrm{mg} /$ per $\mathrm{mL}$. The MFS treatment groups received their MFS supplementation every 4 days until termination. $M t b$ cultures were maintained at $37^{\circ} \mathrm{C}$, with $5 \%$ $\mathrm{CO}_{2}$ until they were terminated at 8 and 15 days post-infection to determine the viability of $M t b . \mathrm{Mtb}$ viability was ascertained by plating the diluted samples on 7H11 agar medium (Hi Media, Santa Maria, CA, USA) enriched with albumin dextrose complex (ADC) (Gemini, West Sacramento, CA, USA).

\subsection{Isolation of Plasma and Monocytes}

Plasma and peripheral blood mononuclear cells (PBMCs) were isolated from the whole blood of T2DM subjects and healthy individuals by Ficoll-Paque (Sigma) density centrifugation. This procedure involves centrifugation of blood layered on Ficoll-Paque at a 1:1 ratio at $1800 \mathrm{rpm}$ for $30 \mathrm{~min}$. Plasma (the top layer) was collected and stored at $-80^{\circ} \mathrm{C}$, while PBMCs (the second layer from the top) were further washed three times with $1 \times$ phosphate-buffered saline (PBS) from Fisher Scientific International Inc. ( $\mathrm{pH} 7.4 \pm 0.1$ ) and then resuspended in RPMI containing L-glutamine and $5 \%$ human AB serum.

\subsection{Induction of Granulomas}

PBMCs were plated on a 24-well tissue culture plate, precoated with $0.001 \%$ poly-L-lysine, and infected with $M t b$ at a multiplicity of infection (MOI) of 0.1:1 bacteria-to-cell ratio (approximately $6 \times 10^{4}$ bacteria were added to $6 \times 10^{5} \mathrm{PBMCs}$ ). Infected cells were maintained at $37^{\circ} \mathrm{C}$ with $5 \%$ $\mathrm{CO}_{2}$ for up to 15 days. Infected PBMCs were then either sham-treated or treated with $0.69 \mathrm{mg}$ of MFS every 4 days until termination. Granulomas were terminated at 8 days and 15 days post-infection to determine the effects of the MFS in altering the granulomatous responses against $M t b$ infection.

\subsection{Cell Termination and Determination of $M t b$ Erdman Survival}

Cell free supernatants from each well were first collected and stored, the cells remaining were then harvested by adding $250 \mu \mathrm{L}$ of ice-cold, sterile $1 \times$ PBS (Sigma, St. Louis, MO, USA) followed by gentle scraping to achieve maximum recovery of cell lysate from the wells. Lysates were then efficaciously vortexed followed by a freeze/thaw cycle in order to ensure sufficient rupture of cells and release of intracellular $M t b$. The collected lysates and supernatants were then diluted as necessary in sterile $1 \times$ PBS (Sigma, St. Louis, MO, USA) and plated on 7H11 agar medium (Hi Media, Santa Maria, CA, USA) enriched with ADC (GEMINI, West Sacramento, CA, USA) and incubated at $37^{\circ} \mathrm{C}$ for 3 weeks in order to evaluate the mycobacterial growth or survival subsequent to MFS treatment by counting the colony forming units (CFUs).

\subsection{Hematoxylin and Eosin Staining}

Granulomas on cover-glasses terminated at 15 days post-infection were fixed with $4 \%$ Paraformaldehyde (PFA) at room temperature for an hour. Fixed granulomas were washed once with 1x PBS and stained with Rapid H\&E (Scientific R\&D Corp, NY, USA) for 2 min and the excess stain was washed away with tap water. The cover glasses were inverted and mounted onto slides with mounting media (HistoChoice, Solon, OH, USA).

\subsection{Quantification of GSH Levels}

Measurement of total and oxidized glutathione was performed using the GSH colorimetric assay kit from Arbor Assays (Ann Arbor, MI, USA). Granuloma lysates were first comprehensively mixed with an equal volume of cold $5 \%$ sulfosalicylic acid (SSA), followed by incubation for $10 \mathrm{~min}$ at $4{ }^{\circ} \mathrm{C}$, and subsequently centrifuged at $14,000 \mathrm{rpm}$ for $10 \mathrm{~min}$. The GSH was thereupon measured in the 
supernatants following the manufacturer's instructions. Reduced GSH (rGSH) was calculated by subtracting the oxidized glutathione disulfide (GSSG) from the total GSH. All measurements were corrected for total protein levels.

\subsection{Quantification of Total Protein Levels}

Total protein was measured with a BCA Protein Assay Kit and performed per user instructions from Thermo Scientific (Rockford, IL, USA).

\subsection{Assay of Cytokines}

Levels of IL-10, IL-12, and IFN- $\gamma$, from the supernatants of THP-1 cells, and granulomas derived from PBMCs of healthy subjects and individuals with T2DM were measured by sandwich ELISA. The assay kits were purchased from eBioscience (San Diego, CA, USA) and performed as per the manufacturers' protocol.

\subsection{Statistical Analysis}

Statistical data analyses were performed using GraphPad Prism version 7 (San Diego, CA, USA). Baseline levels of GSH, rGSH, MDA, IL-6, IL-10, IL-12, IFN- $\gamma$, and TNF- $\alpha$ were compared between healthy individuals and T2DM group using the unpaired $t$-test with Welch correction. Reported values are in means. Statistical significance was determined at ${ }^{*} p<0.05,{ }^{* *} p<0.005,{ }^{* * *} p<0.0005$.

\section{Conclusions}

Collectively, the results demonstrate that MFS treatment strongly influences against Mtb infectivity, including antimycobacterial effects, GSH enrichment, cytokine regulation and augmented granuloma formation. Our results indicated that these benefits can be seen among different cell types including THP-1 macrophages and PBMCs of both healthy participants and immunocompromised individuals with T2DM. The countermeasure effect of MFS treatment against Mtb infectivity in PBMCs from study participants with T2DM was robust but did not achieve the low post-treatment levels attained with the health participants. The cell culture data presented in this article extends the current research of flavonoid's prophylactic potentiality, and suggests that increased flavonoid intake may be attractive adjunctive strategy for managing TB. Although, the in-vivo relevance of data indicating anti-pathogenic activity of flavonoid glycosides and aglycones in cell cultures has not yet been clearly established. The independent antipathogenic effect of the nutrient mix included in the study supplement (vitamin C, caffeine, omega 3 fatty acids) was not measured in this study.

Author Contributions: conceptualization, V.V., D.C.N., M.P.F., and A.S.; methodology, V.V., and D.C.N.; software, R.C., and V.V.; validation, V.V., D.C.N., and G.T.; formal analysis, V.V., D.C.N., R.C., and G.T.; investigation, R.C., H.I., and G.T.; resources, V.V.; data curation, V.V.; writing—original draft preparation, R.C., G.T., H.I., M.G., O.S., S.M., D.C.N., and V.V.; writing-review and editing, D.C.N., R.C., and G.T.; visualization, A.S., D.C.N., and V.V.; supervision, V.V.; project administration, D.C.N., and V.V.; funding acquisition, M.P.F., D.C.N., and V.V.

Funding: This research was funded by Western University if Health Sciences (Office of Vice President for Research and Biotechnology), CA, USA.

Acknowledgments: Our study has been approved by the Institutional Review Board and Institutional Biosafety Committee. We thank all the study subjects for participating in this study.

Conflicts of Interest: The authors declare no conflicts of interest.

\section{References}

1. World Health Organization. Global Tuberculosis Report 2015; World Health Organization: Geneva, Switzerland, 2015.

2. Tacconelli, E.; Carrara, E.; Savoldi, A.; Harbarth, S.; Mendelson, M.; Monnet, D.L.; Pulcini, C.; Kahlmeter, G.; Kluytmans, J.; Carmeli, Y.; et al. Discovery, research, and development of new antibiotics: The WHO priority list of antibiotic-resistant bacteria and tuberculosis. Lancet. Infect. Dis. 2018, 18, 318-327. [CrossRef] 
3. Lönnroth, K.; Roglic, G.; Harries, A.D. Improving tuberculosis prevention and care through addressing the global diabetes epidemic: From evidence to policy and practice. Lancet Diabetes Endo. 2014, 2, 730-739. [CrossRef]

4. World Health Organization. Diabetes. Available online: http://www.who.int/news-room/fact-sheets/ detail/diabetes (accessed on 30 July 2018).

5. Balentine, D.A.; Dwyer, J.T.; Erdman, J.W., Jr.; Ferruzzi, M.G.; Gaine, P.C.; Harnly, J.M.; Kwik-Uribe, C.L. Recommendations on reporting requirements for flavonoids in research. Am. J. Clin. Nutr. 2015, 101, 1113-1125. [CrossRef] [PubMed]

6. Zamora-Ros, R.; Knaze, V.; Rothwell, J.A.; Hémon, B.; Moskal, A.; Overvad, K.; Tjønneland, A.; Kyrø, C.; Fagherazzi, G.; Boutron-Ruault, M.C.; et al. Dietary polyphenol intake in Europe, the European Prospective Investigation into Cancer and Nutrition (EPIC) study. Eur. J. Nutr. 2016, 55, 1359-1375. [CrossRef] [PubMed]

7. Williamson, G.; Kay, C.D.; Crozier, A. The bioavailability, transport, and bioactivity of dietary flavonoids: A review from a historical perspective. Comp. Rev. Food Sci. Food Saf. 2018, 17, 1054-1112. [CrossRef]

8. Cassidy, A.; Rogers, G.; Peterson, J.J.; Dwyer, J.T.; Lin, H.; Jacques, P.F. Higher dietary anthocyanin and flavonol intakes are associated with anti-inflammatory effects in a population of US adults. Am. J. Clin. Nutr. 2015, 102, 172-181. [CrossRef] [PubMed]

9. Somerville, V.S.; Braakhuis, A.J.; Hopkins, W.G. Effect of flavonoids on upper respiratory tract infections and immune function: A systematic review and meta-analysis. Adv. Nutr. 2016, 7, 488-497. [CrossRef] [PubMed]

10. Soh, A.Z.; Pan, A.; Chee, C.B.E.; Wang, Y.T.; Yuan, J.M.; Koh, W.P. Tea drinking and its association with active tuberculosis incidence among middle-aged and elderly adults: The Singapore Chinese Health Study. Nutrients 2017, 9, 544. [CrossRef] [PubMed]

11. Chen, M.; Deng, J.; Li, W.; Lin, D.; Su, C.; Wang, M.; Li, X.; Abuaku, B.K.; Tan, H.; Wen, S.W. Impact of tea drinking upon tuberculosis: A neglected issue. BMC Public Health 2015, 15, 515. [CrossRef] [PubMed]

12. Ahmed, M.; Henson, D.A.; Sanderson, M.C.; Nieman, D.C.; Gillitt, N.D.; Lila, M.A. The protective effects of a polyphenol-enriched protein powder on exercise-induced susceptibility to virus infection. Phytother. Res. 2014, 28, 1829-1836. [CrossRef] [PubMed]

13. Sasikumar, K.; Ghosh, A.R.; Dusthackeer, A. Antimycobacterial potentials of quercetin and rutin against Mycobacterium tuberculosis H37Rv. 3 Biotech 2018, 8, 427. [CrossRef] [PubMed]

14. Anand, P.K.; Kaul, D.; Sharma, M. Green tea polyphenol inhibits Mycobacterium tuberculosis survival within human macrophages. Int. J. Biochem. Cell Biol. 2006, 38, 600-609. [CrossRef] [PubMed]

15. Cialdella-Kam, L.; Nieman, D.C.; Knab, A.M.; Shanely, R.A.; Meaney, M.P.; Jin, F.; Sha, W.; Ghosh, S. A mixed flavonoid-fish oil supplement induces immune-enhancing and anti-inflammatory transcriptomic changes in adult obese and overweight women-a randomized controlled trial. Nutrients 2016, 8, 277. [CrossRef] [PubMed]

16. Mannino, M.H.; Zhu, Z.; Xiao, H.; Bai, Q.; Wakefield, M.R.; Fang, Y. The paradoxical role of IL-10 in immunity and cancer. Cancer Lett. 2015, 367, 103-107. [CrossRef] [PubMed]

17. Allen, M.; Bailey, C.; Cahatol, I.; Dodge, L.; Yim, J.; Kassissa, C.; Luong, J.; Kasko, S.; Pandya, S.; Venketaraman, V. Mechanisms of control of Mycobacterium tuberculosis by NK cells: Role of glutathione. Front. Immunol. 2015, 6, 508. [CrossRef] [PubMed]

18. Teskey, G.; Cao, R.; Islamoglu, H.; Medina, A.; Prasad, C.; Prasad, R.; Sathananthan, A.; Fraix, M.; Subbian, S.; Zhong, L.; et al. The Synergistic Effects of the Glutathione Precursor, NAC and First-Line Antibiotics in the Granulomatous Response Against Mycobacterium tuberculosis. Front. Immunol. 2018, 9, 2069. [CrossRef] [PubMed]

19. Kampmann, B.; Whittaker, E. Immunology of Tuberculosis in Children. In Handbook of Child and Adolescent Tuberculosis; Oxford University Press: Oxford, UK, 2016; pp. 31-42, ISBN 978-0-19-022089-1.

20. Moreira-Teixeira, L.; Sousa, J.; McNab, F.W.; Torrado, E.; Cardoso, F.; Machado, H.; Castro, F.; Cardoso, V.; Gaifem, J.; Wu, X.; et al. Type I IFN inhibits alternative macrophage activation during Mycobacterium tuberculosis infection and leads to enhanced protection in the absence of IFN- $\gamma$ signaling. J. Immunol. 2016, 197, 4714-4726. [CrossRef] [PubMed]

21. Daoud, A.K.; Tayyar, M.A.; Fouda, I.M.; Harfeil, N.A. Effects of diabetes mellitus vs. in vitro hyperglycemia on select immune cell functions. J. Immunotoxicol. 2009, 6, 36-41. [CrossRef] [PubMed] 
22. Nieman, D.C.; Ramamoorthy, S.; Kay, C.D.; Goodman, C.L.; Capps, C.R.; Shue, Z.L.; Heyl, N.; Grace, M.H.; Lila, M.A. Influence of ingesting a flavonoid-rich supplement on the metabolome and concentration of urine phenolics in overweight/obese women. J. Proteome Res. 2017, 16, 2924-2935. [CrossRef] [PubMed]

Sample Availability: Samples of the compounds are not available from the authors.

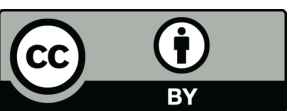

(C) 2019 by the authors. Licensee MDPI, Basel, Switzerland. This article is an open access article distributed under the terms and conditions of the Creative Commons Attribution (CC BY) license (http://creativecommons.org/licenses/by/4.0/). 